K. V. Moore and G. E. Gruen

Idaho Nuclear Corporation

Idaho Falls, Idaho

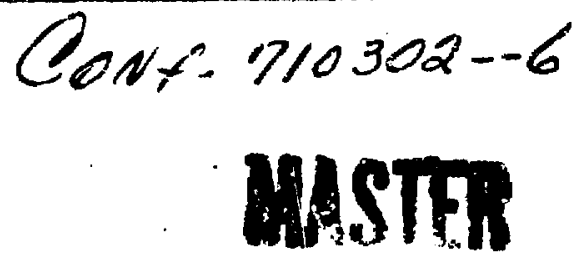

ABSTRACT

As part of a continuing $\in$ ffort in the development of computer codes for reactor: safety analysis, the ASME equations of state for water have been incorporated into RELAP3 as a trial version called RELAP3/ASME. In comparisons to the experimental data from Semiscale Test $820-1$, this trial version gives substantial improvement over RELAP3 in predicting subcosled blowdown pressure histories.

The standard version of RELAP3 is a computer code for analyzing reactor core and loop thermal. hydraulics during blowdown. Continuous calculations from the subcooled portion of the blowdown into the two-phase regicle are at least conceptually permitted. The failure of RELAP3 to correctly predict subcooled pressure histories was traced to inaccurate sonic velocities. Water properties are built-in as tables of pressure versus enthalpy and density. Thermodynamic derivative quantities, such as sonic velocity, are implicit within these tables.

These tables and any dependence on interpolation schemes were eliminated in RELAP3/ASME by incorforating the $1967 \mathrm{ASME}$ equations of state. Each equation, which is defined for particular ranges of pressure and temperature, uses either pressure and temperature or specific volume and temperature as independent variables. Within RELAP3/ASME, a two-dimensional Taylor series expansion is performed on the ASME equations which transforms the independent variables to specific internal energy and specific volume. This complex transformation is performed many times for each volume during a transient calculation and therefore is not efficieni with respect to computer time. Current work is directed at making an efficient production code by developing tables of Taylor coefficients that can be used as explicit equations of state. These techniques and subroutines will need only slight modifications for any choice of independent thermodynamic variables.

\title{
INTRODUCTION
}

As part of a continuing effort in the development of computer codes for reactor safety analysis, the 1967 ASME equations of state for water were incorporated on a trial basis into the RELAP3 code[1,2]. The major purpose of this trial version was to investigate, qualitatively, how another computer form of state properties affects blowdown calculation.

The results reported here are based on this trial version of the RELAP3 code called RELAP3/AGME. No effort was made to make RELAP3/ASME an efficient production tool and in fact, its only purpose was for this study. 
Previous experience with blowdown codes using the nodal volume concept showed deficiency in their ability to predict subcooled decompression to an acceptable accuracy. Although the current physical models and solution techniques used in RELAP 3 can and will be improved in future generations of the code, our main concern herein is to determine what form of state propertios is necessary for Iuture blowdown codes.

\section{EXPERIMENTAI WORK}

Considerable experimental blowdown data exists from the Semiscale series of tests produced by Idaho Nuclear Corporation [3]. This test rig is a small scale mock-up of a pressurized water reactor hydraulic system. The major components are the reactor vessel, steam generator, and primary coolant pump. The general layout of these components and interconnecting piping is shown in Figure 1.

Test 820-1 was chosen as the experimental base for several resons: an uncluttered one-dimensional geometry existed from the auxiliary nozzle through the steam generator (Figure 1); the greatest pressure transients occurred during this test in comparison to other semiscale tests; and the simulated offset shear break presents the greatest probability of significant two-phase conditions behind the initial decompression wave.

Test 820-1 used two blowdown nozzle assemblies to simulate a double-ended break. Simultaneous $100 \%$ pipe area break capability was designed for both nozzles as shown in Figure 1. A crossover pipe connecting the two blowdown pipes allowed some minor flow prior to blowdown for developing the desised isothermal temperature o'istribution throughout the system. A valve in the crossover pipe was closed just prior to initiation of the blowdown.

Initial conditions for Test $820-1$ were a temperature of $588^{\circ} \mathrm{F}$, a pressure of $2263 \mathrm{psia}$, and no system flow. The breaks were sized at $100 \%$ of the $0.09 \mathrm{ft}^{2}$ blowdown pipe areas for a double--ended-break blowdown having a total break area of $0.18 \mathrm{ft}^{2}$.

The method of rupture initiation for Semiscale Test 820-1. was underpressurizatjon of the space jetween two rupture disks located in the blowdown nozzle ussembly. For this method, the urstream disk ruptures first and starts a decompression into the system. At this time a pressure wave is moving out of the system and hits the downstream disk, starting a compression wave into the system. About 1 msec later the downstream disk ruptures and starts a second decompression wave into the system. Jinus, the initial primary decompression process is composed of two parts that are dependent on the individuai disk characteristics [4].

The decompression wave traveling into this system from the auxiliary rozzle is relatively simple although the acoustical reflections from the steam generator (24 feet from the auxiliary nozzle) produce a complex pressure transient. As shown in Figure 2, the experimental pressure record of P5 (which is located 1.07 It below the steam generator) begins to fall at 8 milliseconds which is the pressure wave propagation time for 24 feet at $3000 \mathrm{ft} / \mathrm{sec}$. Between 8 and 10 milliseconds, several minor pressure fluctuations are superimposed on the general decompressions. These are attributed to the two-part primary decompression wave generated by the rupture device and to reflections from the tubes within the steam generator. The pressure rise, which begins at 12 milliseconds, is attributed to the reflection from the upper plenum of the steam generator. 
RELAP3 is a multivolume compressible hydrodynamics and heat transfer code desigined to analyze the transient phenomena of a reactor blowdown. In RELAP3, pressures, flows, temperatures, and other hydraulic related quantites are calculated as averages over mathematical control volumes. The choice of volume size is entirely a user option. To simulate Test 820-1 with a minimum of extraneous complications such as the stability associated with unequal volume sizes, the piping from the auxiliary nozzle to a location beyond $P 6$ was divided into 69 volumes each one-half foot long.

Figure 3 is the pressure history for location P5 as calculated from the published version of RELAP3. In general, the results are unacceptable. The arrival time of the decompression wave is about 2 milliseconds early, which suggests an erroneous sonic velocity. Further transient detail has at best only a passing resemblance to the experimental pressure trace of P5. Plots of other RELAP3 volume pressures gave a hint that the major error might be due to the steam tables contained within the code.

Water properties are contained within RELAP3 as tables of pressure vs. enthalpy and density. In general, these tables are constructed at 24 different pressures from .I psia to 3200 psia and at each pressure there are 13 entries of density and enthalpy for the liquid region and five additional entries for the super heat region to $5600^{\circ} \mathrm{F}$.

Sonic velocity, which thermodynamically is defined as $\sqrt{\left(\frac{\partial_{p}}{\partial_{p}}\right)}$ for single phase fluids, is contained implicitly within the tables. In the pressure range of interest for this problem, the tables are spaced at even increments: 1200, 1400, $1600,1800 \ldots 2400$ psia. Since the interpolation technique involves a doublelinear interpolation, first on pressure, then on density and enthalpy, the sonic velocity produced by these tables can change discontinuously across pressure entry points as shown in Figure 4. The curve labeled RELAP3 is density vs. pressure for a constant internal energy as determined from the water property tables. The curve labeled ASME is density vs. pressure for a constant temperature as computed from the ASME equations. The slope of the ASME curve is smoothly varying as contrasted to the discontinuities in the slope of the RELAP3 curve.

This resulting discontinuity in sonic velocity with RELAP3 was thought to ke the cause of the false pressure reflections during a calculated transient. To test this idea, the ASME equations were adapted to RELAP3.

\section{REILAP3/ASME}

The combination of KELAP3 and the ASME equations, named RELAP3/ASME formed an inefficient long-running code which was useful only for these calculations. Within RELAP3/ASME, a two-dimensional Taylor series expansion is performed on the ASME equations which transforms the independent variables to specific internal energy and specific volume. This transformation is performed many times for each volume during a transient calculation and therefore is very inefficient with respect to computer time. Previous work with the ASME equations showed that the derivatives were smooth and continuous to a very high degree except at boundaries where the form of the ASME equations change from a Gibbs function to a Helmholtz function. These narrow discontinuities occur at 2398.2 psia and hence have no effect on calculations for Test 820-1.

Figure 5 is the pressure history of P5 as calculated by RELAP3/ASME and Figure 6 Is an overlay of this calculated curve and the experimental pressure trace. The 
agreement is much superior in comparison to the RELAP3 calculation. The initial decompression wave at $P 5$ is calculated 'to occur within .5 milliseconds of the experimental value and the first reflection from the steam generator starts 1.5 milliseconds later, which is the round-trip time from P5 to the steam generator. Approximately $3.5 \mathrm{milliseconds}$ after the first decompression wave passes 15 , the broad recompression wave, which is reflected from the steam generator upper plenum arrives at P5. Qualitatively, this slope agrees with the experimental trace. The high frequency oscillation superimposed on the calculated pressure is due to an irstability associated with the volume size in the REIAP3/ASME calculations.

\section{CONCLUSIONS}

Since the only change between RELAP3 and RELAP3/ASME was in the state property formulations, the calculated differences between these codes is due to the inaccuracies in the standard REIAP3 state properties and the equivalent derivatives. Subsequent work not reported here has shown that this type of error is also present in two-phase transients as well as the subcooled phase although the errors are not nearly so pronounced.

Our conclusions are that transient hydraulic codes intended for compressible fluid dynamic analysis must contain resonably accurate state properties including smoothly varying derivatives of the state properties.

\section{CURRENT WORK}

A state property package based on sets of Taylor coefficients is currently being developed, and the user will have a limited choice of independent thermodynamic variables. Current designs include specific energy and specific volume ( $u, v$ ), pressure and internal energy $(P, u)$, and enthalpy and specific volume $(h, v)$. This package is complete for the two-phase region and is nearing completion for the single-phase regions. As designed, this package is an independent module for general use. The execution rate is approximately 10,000 independent state solutions per 1.5 minutes of $360 / 75$ CPU time. This speed is comparable to the published REIAP3 code and considerably faster (by severel orders of magnitude) than REIAAP3/ASME.

\section{REFERENCES}

1. C. A. Meyer, R. B. McClintock, G. J. Silvestri, R. C. Spencer, Jr., "Thermodynamic and Transport Properties of Steam", The American Society of Mechanical Engineers, (1967)

2. W. J. Rettig, G. A. Jayne, K. V. Moore, C. E. Slater, M. L. Uptmor, RELAP3-A Computer Program for Reactor Blowdown Analysis, IN-1321, (June 1970)

3. H. H. Heiselmann, D. J. Olson, J. F. Whitbeck, Semiscaje and Emergency Core Cooling (ECC) Project Test Report--Tests 803, Through 820, IN-1404, (October 1970)

4. G. E. Gruen, WHAM Prediction of Semiscale Test Results, IN-143i, (October 1970) 


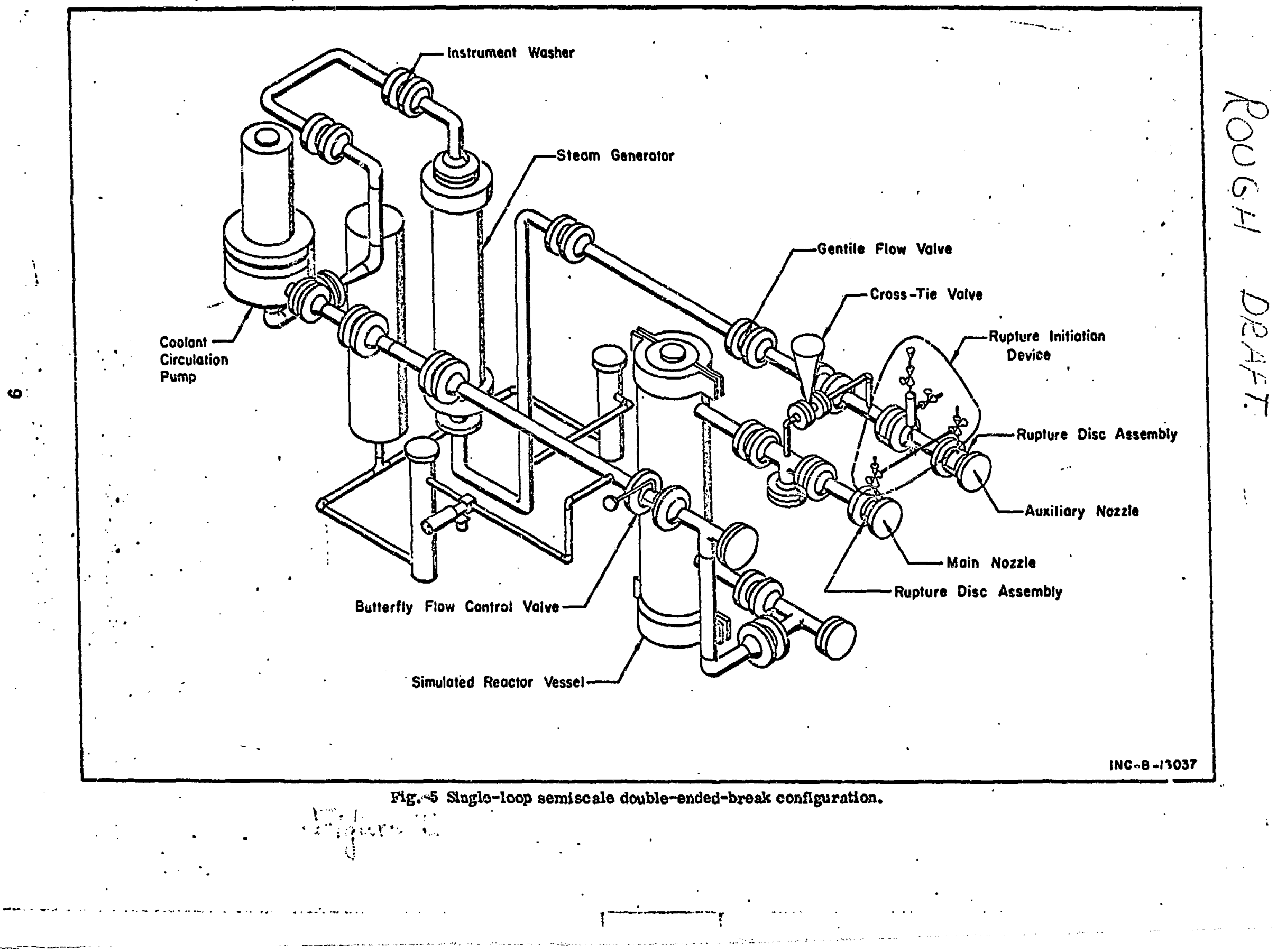




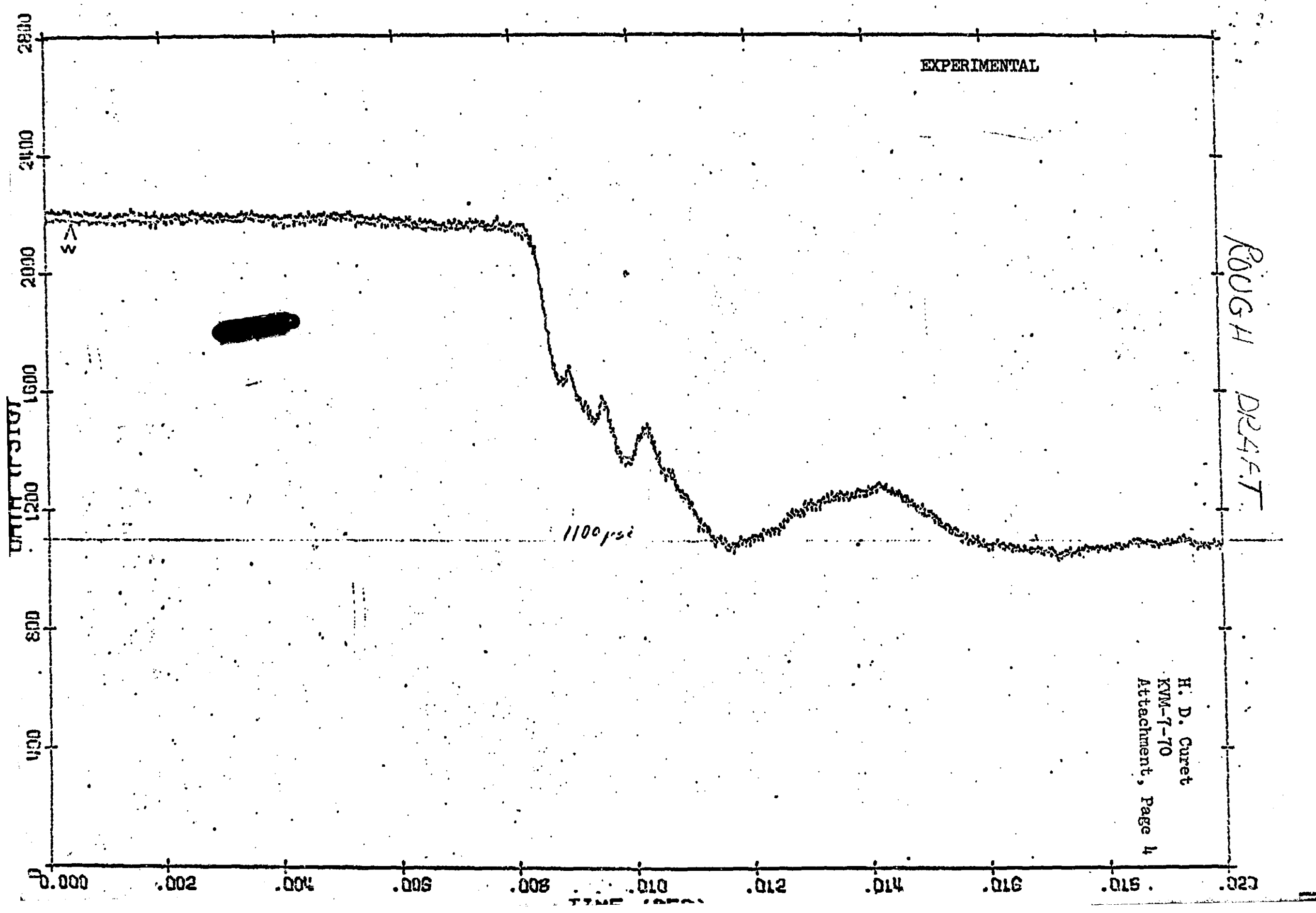




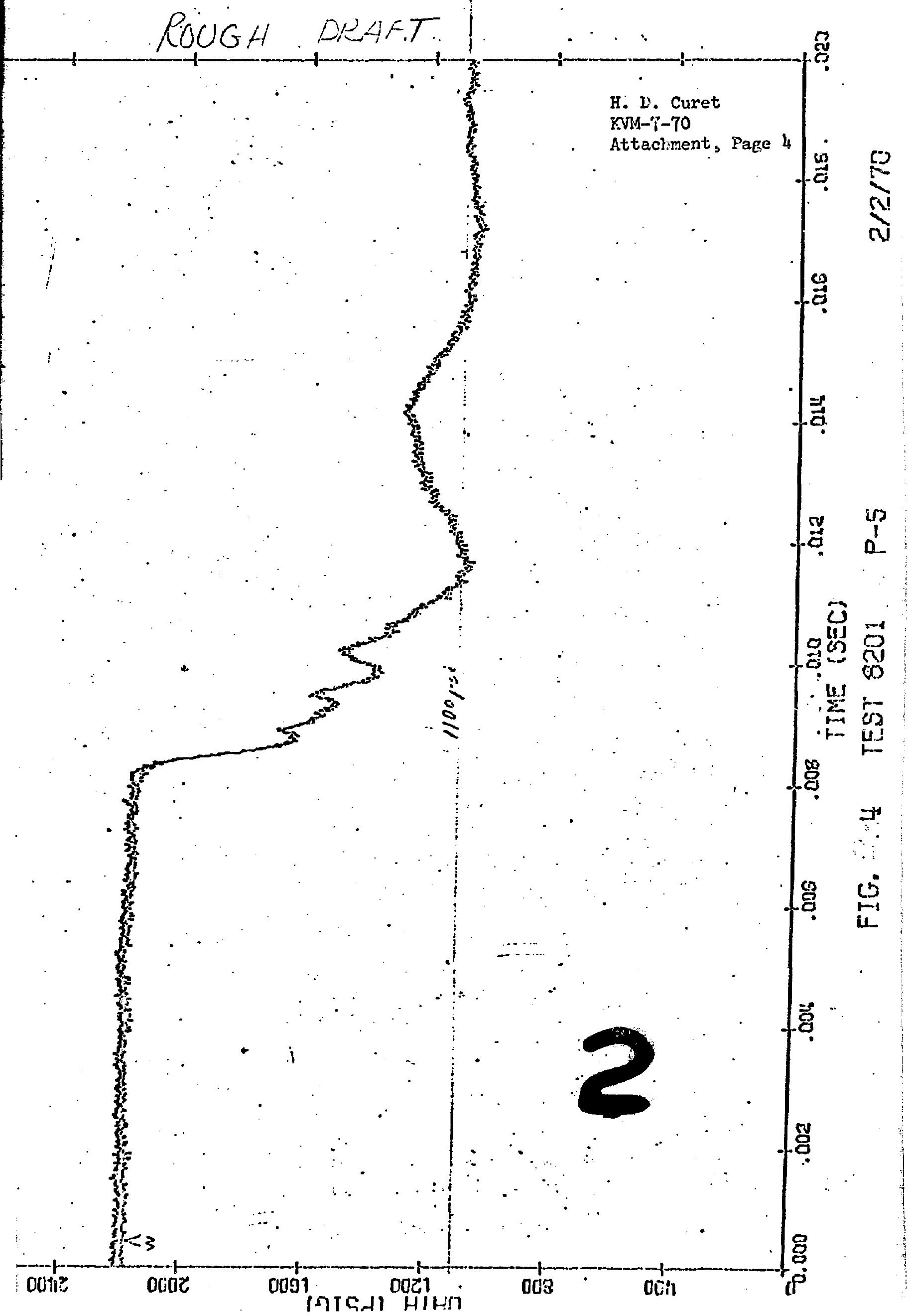




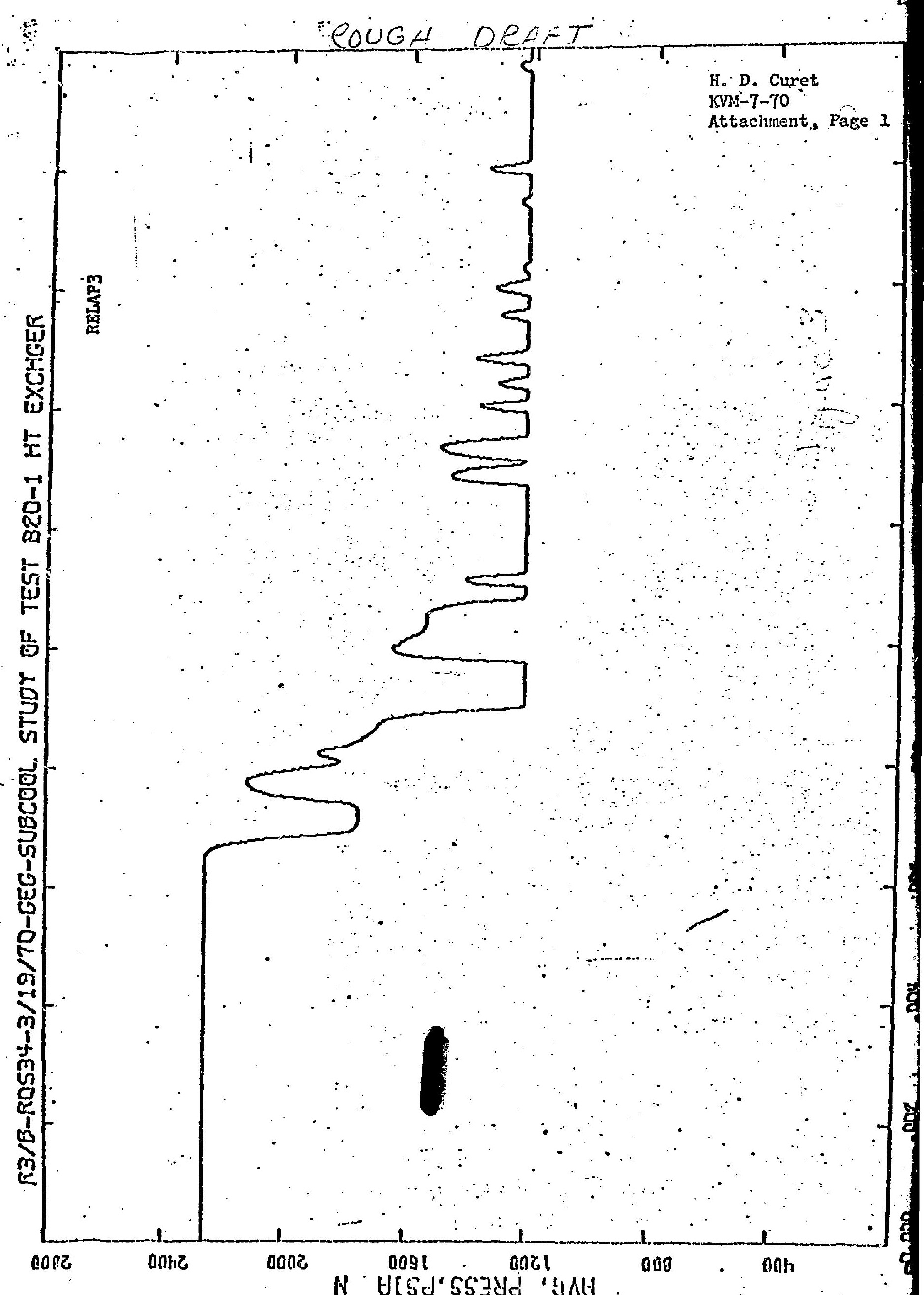





\section{ROUGH DKAFT}

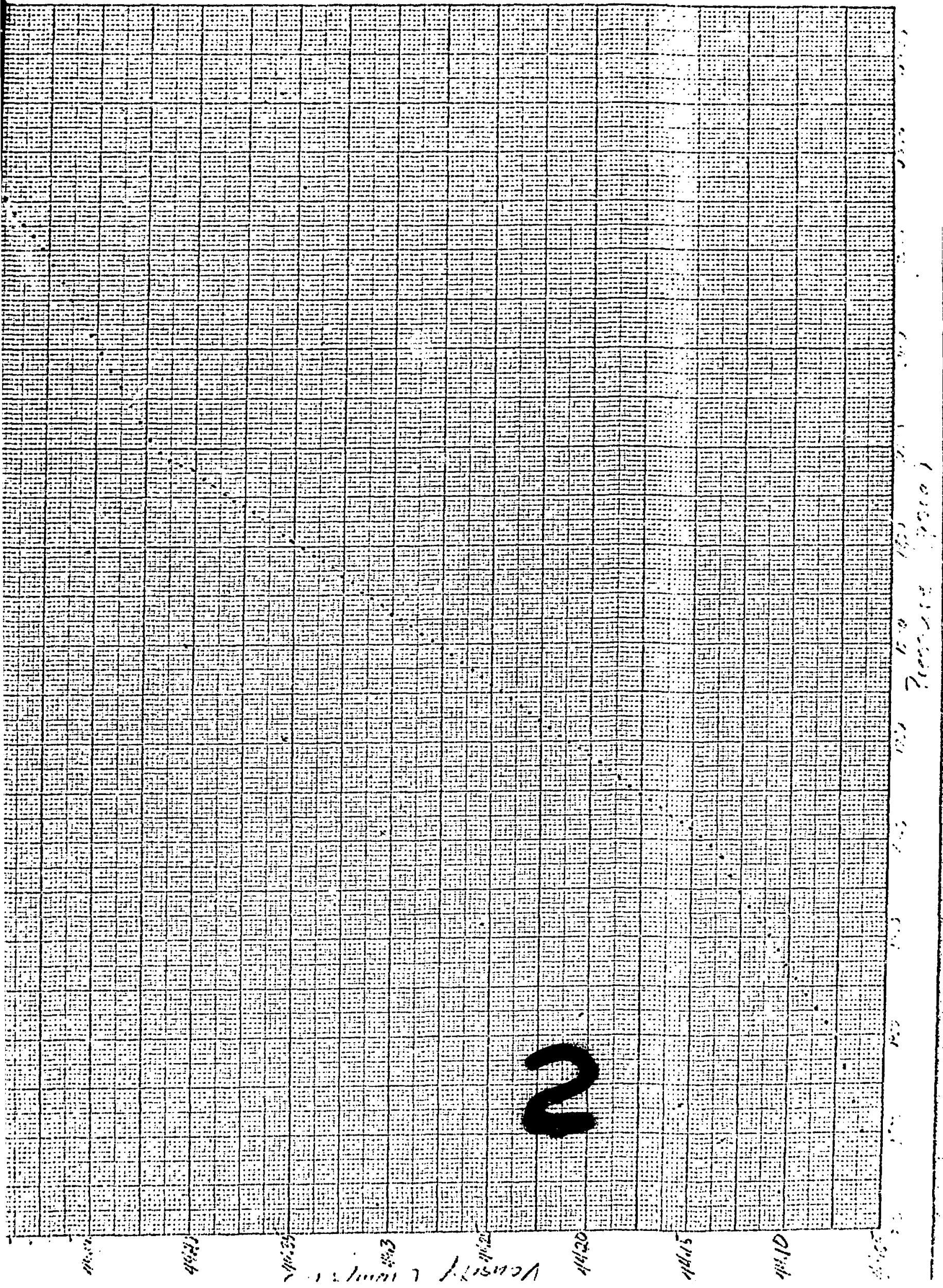


<smiles>CCCSCC</smiles> 


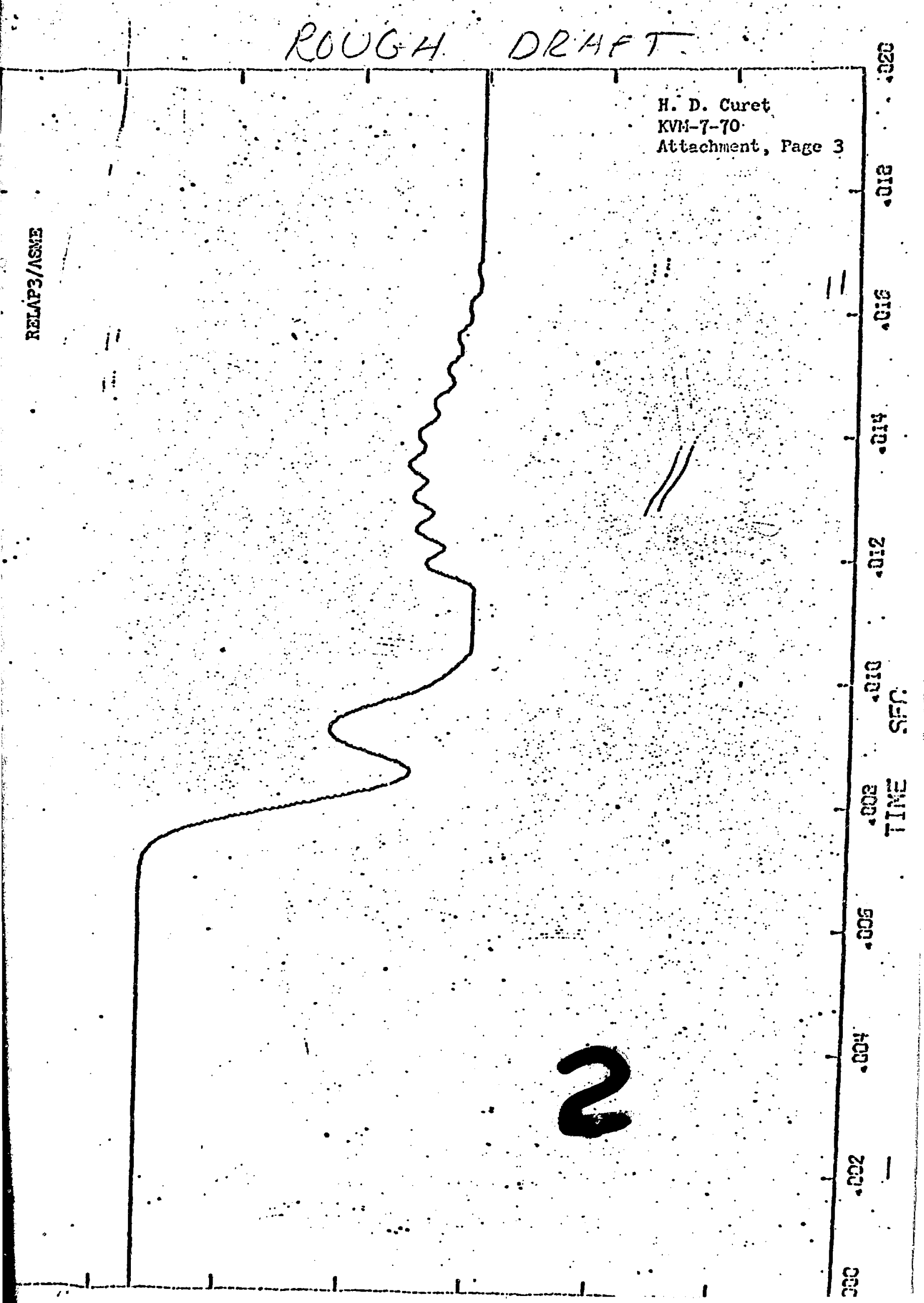




\section{ROUGA DRAFT}

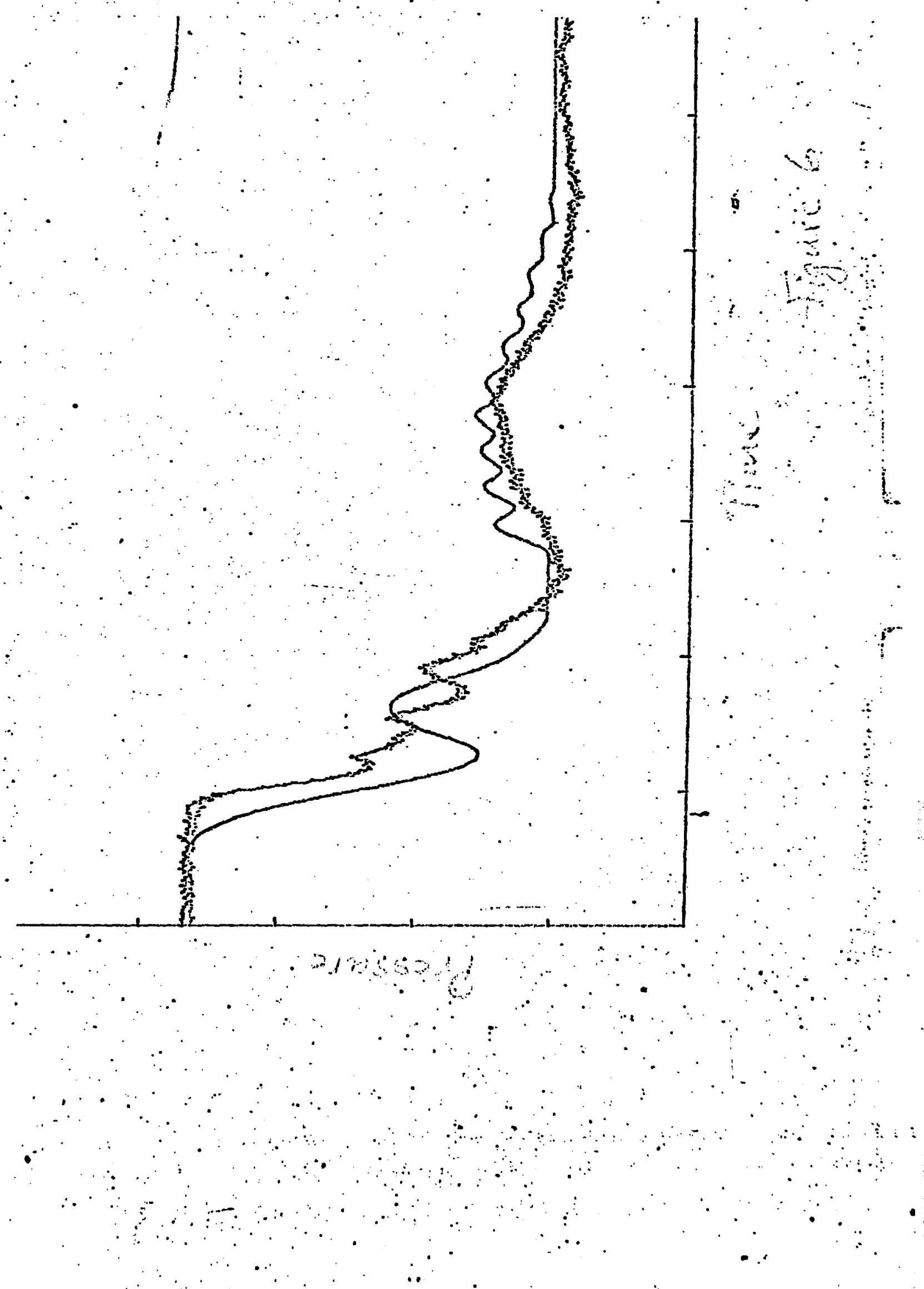

